\title{
New approach to twisted $q$-Bernoulli polynomials
}

\author{
Daeyeoul Kim', Ja Kyung Koo² and Yoon Kyung Park ${ }^{3 *}$
}

"Correspondence:

ykpark@math.kaist.ac.kr

${ }^{3}$ School of Mathematics, Korea

Institute for Advanced Study (KIAS),

85 Hoegiro, Dongdaemun-gu,

Seoul, 130-722, South Korea

Full list of author information is

available at the end of the article

\begin{abstract}
By using the theory of basic hypergeometric series, we present some formulas for $q$-consecutive integers, and we find certain new identities for twisted $q$-Bernoulli polynomials and $q$-consecutive integers (Simsek in Adv. Stud. Contemp. Math. 16(2):251-278, 2008).
\end{abstract}

MSC: $11 \mathrm{~B} 68 ; 05 \mathrm{~A} 30$

Keywords: q-consecutive integer; twisted q-Bernoulli polynomial

\section{Introduction}

The classical Bernoulli polynomials $B_{n}(x)$ and the Euler polynomials $E_{n}(x)$ are usually defined by the generating functions

$$
\begin{aligned}
\frac{t e^{x t}}{e^{t}-1}=\sum_{n=0}^{\infty} B_{n}(x) \frac{t^{n}}{n !}, & |t|<2 \pi \quad \text { and } \\
\frac{2 e^{x t}}{e^{t}+1}=\sum_{n=0}^{\infty} E_{n}(x) \frac{t^{n}}{n !}, & |t|<\pi,
\end{aligned}
$$

respectively. In addition, the Bernoulli numbers are given by $B_{n}:=B_{n}(0)$ for $n \geq 1$. Recently, the Bernoulli polynomials and Bernoulli numbers have gained considerable significance in the fields of physics and mathematics [1-4]. For example, Kim [3] defined a new $q$-analogy of the Bernoulli polynomials and Bernoulli numbers, and he deduced some important relations between them. Moreover, $q$-analogues have been investigated in the study of quantum groups and $q$-deformed superalgebras [1]. The connection here is similar, in that much the string theory is set in the language of Riemann surfaces, resulting in connections with elliptic curves, which in turn relate to $q$-series. A $q$-analogue is an identity for a $q$-series that returns a known result in the 'bosonic' limit (in contrast to the conventional 'fermionic' limit $q \rightarrow-1$ ) as $q \rightarrow 1$ (from inside the complex unit circle in most situations). In addition to the widely used $q$-series, we have $q$-numbers, $q$-factorials, and $q$-binomial coefficients. A $q$-number is obtained by observing $\lim _{q \rightarrow 1} \frac{1-q^{n}}{1-q}=n$. Thus, we define a $q$-number as $[n]_{q}=\frac{1-q^{n}}{1-q}$. Accordingly, one can define the $q$-analogue of the factorial, namely, $q$-factorial, as

$$
\begin{aligned}
{[n]_{q} ! } & =[1]_{q}[2]_{q} \cdots[n-1]_{q}[n]_{q} \\
& =\frac{1-q}{1-q} \frac{1-q^{2}}{1-q} \cdots \frac{1-q^{n}}{1-q}=1 \cdot(1+q) \cdots\left(1+q+\cdots+q^{n-2}\right)\left(1+q+\cdots+q^{n-1}\right) .
\end{aligned}
$$

(02013 Kim et al.; licensee Springer. This is an Open Access article distributed under the terms of the Creative Commons Attribution License (http://creativecommons.org/licenses/by/2.0), which permits unrestricted use, distribution, and reproduction in any medium, provided the original work is properly cited. 
Using this notation, we can define the $q$-binomial coefficients, also known as Gaussian coefficients, by

$$
\left[\begin{array}{l}
n \\
k
\end{array}\right]_{q}=\frac{[n]_{q} !}{[n-k]_{q} ![k]_{q} !} .
$$

Furthermore, the $q$-Bernoulli polynomials $\beta_{n, q}(x)$ and the $q$-Bernoulli numbers $\beta_{n, q}$ can be defined in terms of the generating function $F_{t}(x, q)$ as follows [5]:

$$
F_{t}(x, q)=e^{\frac{1}{1-t}} \frac{t-1}{\log t}-q \sum_{n=0}^{\infty} t^{n+x} e^{[x+n] t q}=\sum_{n=0}^{\infty} \frac{\beta_{n, t}(x)}{n !} q^{n}, \quad|q|<1,|t|<1 .
$$

Kim [6] established an interesting relation between Bernoulli numbers and $q$-integers, that is,

$$
\int_{0}^{n} \beta_{l, q} d[x]_{q}=\frac{1}{l+1}\left(\beta_{l+1, q}(k)-\beta_{l+1, q}\right) .
$$

In addition, Kim [7, Theorem 1], Kim and Lee [8, Lemma 2.1] derived the relations between the Euler polynomials $E_{n}^{(r)}(x)$ of order $r$ using the alternating sum of powers of consecutive integers $T_{k}(n)$. Here, $T_{k}(n)=\sum_{i=0}^{n}(-1)^{r} l^{k}$ and $E_{n}^{(r)}(x)$ is defined as

$$
\left(\frac{2}{e^{t}+1}\right)^{r} e^{x t}=\sum_{n=0}^{\infty} E_{n}^{(r)}(x) \frac{t^{n}}{n !}
$$

Simsek constructed twisted Bernoulli polynomials together with twisted Bernoulli numbers and obtained analytic properties of twisted $L$-functions $[9,10]$. Further, he defined generating functions of the twisted $q$-Bernoulli numbers and polynomials [9]. In a complex case, the generating function of twisted $q$-Bernoulli numbers $f_{q, \omega}(t)$ and a $q$-analogue of the Hurwitz zeta function $f_{\omega}(t, x, q)$ are given by

$$
\begin{aligned}
& f_{q, \omega}(t)=\sum_{l=0}^{\infty} B_{l, \omega}^{*}(q) \frac{t^{l}}{l !} \text { and } \\
& f_{\omega}(t, x, q)=e^{-t[x]_{q}} f_{q, \omega}(t)=\sum_{l=0}^{\infty} B_{l, \omega}^{*}(q, x) \frac{t^{l}}{l !},
\end{aligned}
$$

where $q \in \mathbb{C}$ with $|q|<1$, and $\omega$ is the $r$ th root of 1 . In a complex case, the generating function of twisted $q$-Bernoulli numbers $f_{q, \omega}(t)$ and a $q$-analogue of the Hurwitz zeta function $f_{\omega}(t, x, q)$ are given by

$$
\begin{aligned}
& f_{q, \omega}(t)=\sum_{l=0}^{\infty} B_{l, \omega}^{*}(q) \frac{t^{l}}{l !} \text { and } \\
& f_{\omega}(t, x, q)=e^{-t[x] q} f_{q, \omega}(t)=\sum_{l=0}^{\infty} B_{l, \omega}^{*}(q, x) \frac{t^{l}}{l !},
\end{aligned}
$$


where $q \in \mathbb{C}$ with $|q|<1$, and $\omega$ is the $r$ th root of 1 . Simsek [9] then derived the identities

$$
\begin{aligned}
& B_{l, \omega}^{*}(q)=(-1)^{l} l \sum_{n=0}^{\infty} \omega^{-n} q^{-l n}[n]_{q}^{l-1}, \\
& \zeta_{\omega, q}(1-l)=\frac{(-1)^{l+1}}{l} B_{l, \omega}^{*}(q), \\
& B_{l, \omega}^{*}(x, q)=-(-1)^{l} l \sum_{n=1}^{\infty} \omega^{-n} q^{-l n}[n+x]_{q}^{l-1}, \\
& \zeta_{\omega, q}(1-l, x)=\frac{(-1)^{l+1}}{l} B_{l, \omega}^{*}(x, q),
\end{aligned}
$$

where $\zeta_{\omega, q}(s)=\sum_{n=1}^{\infty} \frac{\omega^{-n} q^{-n}}{\left(q^{-n}[n] q\right)}, \zeta_{\omega, q}(s, x)=\sum_{n=0}^{\infty} \frac{\omega^{-n} q^{-n}}{\left(q^{-n}[n+x] q\right)}$, and $x$ is a natural number. In this paper, we first study relations among $q$-consecutive integers, $q$-Bernoulli numbers, and $q$-Euler numbers.

In 1631, Faulhaber [11] evaluated the sums of powers of consecutive integers $1^{k}+\cdots+n^{k}$ up to $k=17$. Further, in 1993, Knuth [12] presented an insightful alternative account of Faulhaber's work. Several mathematicians further considered the problems of $q$-analogues of such sums of powers $[7-9,13]$. On the basis of Bernoulli's concept, Kim derived a $q$ analogue of the sums of powers of consecutive integers, by setting

$$
\begin{aligned}
& T_{l, q^{h}}(n)=\sum_{k=0}^{n-1}[k]_{q}^{l} q^{h k}, \\
& T_{l, t}=\sum_{k=0}^{\infty}[k]_{q}^{l} t^{k-1}
\end{aligned}
$$

and

$$
\begin{aligned}
S_{l, n}(q) & =\sum_{k=1}^{n} \frac{1-q^{2 k}}{1-q^{2}}\left(\frac{1-q^{k}}{1-q}\right)^{l-1} q^{\frac{(l+1)(n-k)}{2}} \\
& =\sum_{k=1}^{n}[k]_{q}^{l} \frac{1+q^{k}}{1+q} q^{\frac{(l+1)(n-k)}{2}} \\
& =\sum_{k=0}^{n-1}[k+1]_{q}^{l} \frac{1+q^{k+1}}{1+q} q^{\frac{(l+1)(n-k-1)}{2}}
\end{aligned}
$$

with $s \in \mathbb{C}$ and $|s|<1$.

In Section 2, we recall some necessary identities for basic hypergeometric series [14]. Further, we obtain a generalization of Proposition 2.1, and accordingly, we obtain $q$ consecutive integers for $\sum_{k=0}^{n-1}[k]_{q} q^{k}$. These new results are similar to the ones presented in some other studies [7-9] and [13].

In Section 3, we derive a formula for $S_{2, n}$ and $T_{2, q}(n)$ by using a property of basic hypergeometric series, such as

$$
\sum_{k=0}^{\infty} \frac{(a q ; q)_{k}(c q ; q)_{k}}{(q ; q)_{k}(b q ; q)_{k}} t^{k}=\frac{(a q ; q)_{\infty}(c t q ; q)_{\infty}}{(b q ; q)_{\infty}(t ; q)_{\infty}} \sum_{k=0}^{\infty} \frac{(b / a ; q)_{k}(t ; q)_{k}}{(q ; q)_{k}(c t q ; q)_{k}} a^{k} q^{k}
$$


The $q$-analogue Eulerian numbers are defined as [15]:

$$
\begin{aligned}
& E_{m, q}=\lim _{N \rightarrow \infty} \frac{1}{\left[p^{N}\right]_{-q}} \sum_{l=0}^{p^{N}-1}[l]_{q}^{m}(-q)^{l} \text { and } \\
& E_{n, q}(x)=\sum_{l=0}^{n}\left(\begin{array}{l}
n \\
l
\end{array}\right) q^{l x} E_{l, q}[x]_{q}^{n-l} .
\end{aligned}
$$

For these, we establish certain new identities by utilizing basic hypergeometric series, which differ from Bernoulli numbers and polynomials constructed by Kim et al. [16, 17] as follows:

$$
(-1)^{n+1} q^{n} E_{1, q}(n)+E_{1, q}= \begin{cases}{[n]_{q}-[2]_{q}^{2} \frac{[2 n]_{q}}{[4]_{q}}} & \text { if } n \text { is even, } \\ {[\infty]_{q}\left(\frac{[2 n]_{q}}{[n]_{q}}-\frac{[2]_{q}^{2}[4 n]_{q}}{[4]_{q}[2 n]_{q}}\right)} & \text { otherwise }\end{cases}
$$

and

$$
(-1)^{n+1} q^{n} E_{2, q}(n)+E_{2, q}= \begin{cases}{[\infty]_{q}\left([n]_{q}-2 \frac{[2]_{q}^{2}[2 n]_{q}}{[4]_{q}}+\frac{[2]_{q}[3]_{q}[3 n]_{q}}{[6]_{q}}\right)} & \text { if } n \text { is even } \\ {[\infty]_{q}^{2}\left(\frac{[2 n]_{q}}{[n]_{q}}-2 \frac{[2]_{q}^{2}[4 n]_{q}}{[4]_{q}[2 n]_{q}}+\frac{[2]_{q}[3]_{q}[6 n]_{q}}{[6]_{q}[3 n]_{q}}\right)} & \text { otherwise. }\end{cases}
$$

Here, we note that these are related to $T_{l, q^{h}}(n)$.

In Section 4, we deduce recursive formulas from Lemma 4.1 for basic hypergeometric series. More precisely, let $S_{l}(t)=\sum_{k=0}^{\infty} \frac{\left(q^{2} ; q\right)_{k}^{l}}{(q ; q)_{k}^{l}} t^{k}$. Then, we derive the recursive formulas

$$
\begin{aligned}
S_{l+1}(t) & =\frac{1}{1-q}\left(S_{l}(t)-q S_{l}(t q)\right) \\
& =\frac{1}{(1-q)^{l}} \sum_{k=0}^{l+1}\left(\begin{array}{c}
l+1 \\
k
\end{array}\right) S_{0}\left(t q^{k}\right)(-q)^{k} .
\end{aligned}
$$

Using these identities, we obtain a formula for $\sum_{k=0}^{n-1}[k]^{l} q^{k}$, and we present relations between $q$-Bernoulli numbers and $q$-consecutive integers, which are related to (S1)-(S4). Lastly, the rank of partition is defined as the difference between its largest part and the number of its parts. The number of partitions of $n$ with the rank $r$ would be denoted by $P_{r}(n)$. We use the convention $P_{0}(0)=1, P_{r}(n)=0$ for $r \neq 0, n \leq 0$ and $r=0, n<0$. Here, for the sake of convenience, we define

$$
\begin{aligned}
& C_{1}(t ; q)=1 \\
& C_{l}(t ; q)=\frac{1}{1-q}\left(C_{l-1}(t ; q)\left(1-t q^{l}\right)-q C_{l-1}(t q ; q)(1-t)\right) .
\end{aligned}
$$

Then, these are related to $P_{r}(n)$ by the following identity (Remark 4.13):

$$
\sum_{l=0}^{\infty} \frac{T_{l, \frac{q}{u}}}{C_{l}\left(u^{-1} q ; q\right)} u^{l} q^{l+1}=\sum_{r=-\infty}^{\infty} \sum_{n=1}^{\infty} P_{r}(n) u^{r} q^{n}
$$


with $|q|^{2}<|u|<1$. Finally, we shall relate through Theorem 4.7 and Remark 4.14, $q$ Bernoulli polynomials with the third-order mock theta functions introduced by Ramanujan.

Throughout this paper, we adopt the following notations:

- $[k]_{t}=\frac{1-t^{k}}{1-t}$.

- $[\infty]_{t}=\frac{1}{1-t}$.

- $S_{m, n}(q)=\sum_{k=1}^{n} \frac{1-q^{2 k}}{1-q^{2}}\left(\frac{1-q^{k}}{1-q}\right)^{m-1} q^{\frac{m+1}{2}(n-k)}$.

- $T_{l, q^{h}}(n)=\sum_{k=0}^{n-1}[k]_{q}^{l} q^{h k}$.

- $(a ; q)_{n}=(1-a)(1-a q)\left(1-a q^{2}\right) \cdots\left(1-a q^{n-1}\right)$.

- $(a ; q)_{\infty}=\prod_{n=0}^{\infty}\left(1-a q^{n}\right)$.

- $(a ; q)_{0}=1$.

- $\left[\begin{array}{l}m \\ k\end{array}\right]_{q}= \begin{cases}\frac{(q ; q) m}{(q ; q) ;(q ; q)} m-k & \text { if } 0 \leq k \leq m, \\ 0 & \text { otherwise. }\end{cases}$

- $\omega$ : the $r$ th root of unity.

- $F(a, b ; t):=F(a, b ; t: q)=1+\sum_{n=1}^{\infty} \frac{(1-a q)\left(1-a q^{2}\right) \ldots\left(1-a q^{n}\right)}{(1-b q)\left(1-b q^{2}\right) \cdots\left(1-b q^{n}\right)} t^{n}=\sum_{n=0}^{\infty} \frac{(a q ; q)_{n}}{(b q ; q) n} t^{n}$.

\section{Identities of basic hypergeometric series and $\sum_{k=0}^{n-1}[k]_{q} q^{k}$}

In this section, we investigate some identities of basic hypergeometric series. To this end, we refer to [14]. Now, we consider the series defined by

$$
F(a, b ; t: q)=1+\sum_{n=1}^{\infty} \frac{(1-a q)\left(1-a q^{2}\right) \cdots\left(1-a q^{n}\right)}{(1-b q)\left(1-b q^{2}\right) \cdots\left(1-b q^{n}\right)} t^{n}
$$

Fine presented many interesting properties in his book; the following identity represents one such property:

$$
F(a, b ; t: q)=\frac{1-b}{1-t}+\frac{b-a t q}{1-t} F(a, b ; t q: q) .
$$

Throughout this paper, $q$ denotes a fixed complex number of absolute value less than 1 , so that we may write $q=\exp (\pi i \tau)$, where $\tau$ is a complex number with a positive imaginary part. We use $q^{c}$ to denote $\exp (c \pi i \tau)$. The partial product $(a q ; q)_{n}$ converges for all values of $a$, as may be easily seen from the absolute convergence of $\sum q^{n}$. Hence, if $b$ is not one of the values $q^{-1}, q^{-2}, \ldots$, the coefficients $\frac{(a q ; q)_{n}}{(b q ;)_{n}}$ are bounded, and the series (2.1) converges for all $t$ inside the unit circle, and represents an analytic function therein. Hence, the function on the right-hand of (2.2) is regular in the domain $|t|<|q|^{-1}$, except for a simple pole at $t=1$. Therefore, we obtain the continuation of $F$ to a larger circle. Then, it is easy to apply (2.2) again to the continuation of $F$ to the circle $|t|<|q|^{-2}$, and thus, we conclude that for $b \neq q^{-n}, n>1$, the only possible singularities of $F$ occur at the points $t=q^{-n}(n \geq 0)$, which are simple poles in general. As a function of $b, F$ is regular, except possibly at the simple poles $b=q^{-n}(g \geq 1)$, provided that $b$ and $t$ do not have one of the singular values mentioned above. First, we derive Theorem 2.2 by generalizing the following proposition.

Proposition 2.1 For the complex number $q$ and $t$ with $|q|<1$, we have

$$
\sum_{m=0}^{\infty} \frac{(a q ; q)_{2 m}(b q ; q)_{m}}{(a q ; q)_{m}(q ; q)_{m}} t^{m}=\frac{(b t q ; q)_{\infty}}{(t ; q)_{\infty}} \sum_{k=0}^{\infty} \frac{(b q ; q)_{k}(t ; q)_{k}}{(q ; q)_{k}(b t q ; q)_{2 k}}(-a t)^{k} q^{3 k^{2}+k}
$$


Proof Equation (25.96) in [14].

Theorem 2.2 For complex numbers $q, t$ with $|q|<1$ and an integer $l \geq 0$, we get

$$
\sum_{m=0}^{\infty} \frac{(a q ; q)_{(l+1) m}(b q ; q)_{m}}{(a q ; q)_{l m}(q ; q)_{m}} t^{m}=\frac{(b t q ; q)_{\infty}}{(t ; q)_{\infty}} \sum_{k=0}^{\infty} \frac{(b q ; q)_{k}(t ; q)_{l k}}{(q ; q)_{k}(b t q ; q)_{(l+1) k}}(-a t)^{k} q^{\frac{(2 l+1) k^{2}+k}{2}}
$$

To prove this, we need some identities from [14].

Lemma 2.3 (1) For a nonnegative integer $N$,

$$
(t q ; q)_{N}=\sum_{k=0}^{N}\left[\begin{array}{c}
N \\
k
\end{array}\right]_{q}(-t)^{k} q^{\frac{k^{2}+k}{2}} .
$$

It is an analogue of the binomial series, to which one can reduce termwise with $q=1$.

(2)

$$
F(a, 1 ; t: q)=\sum_{n=0}^{\infty} \frac{(a q ; q)_{n}}{(q ; q)_{n}} t^{n}=\frac{(a t q ; q)_{\infty}}{(t ; q)_{\infty}}
$$

(3)

$$
(a q ; q)_{\infty}=\sum_{k=0}^{\infty} \frac{(-a)^{k}}{(q ; q)_{k}} q^{\frac{k^{2}+k}{2}} .
$$

Proof Equations (6.23), (6.2), and (12.44) in [14], respectively.

Proof of Theorem 2.2 We start with the left-hand side in our assertion:

$$
\sum_{m=0}^{\infty} \frac{(a q ; q)_{(l+1) m}(b q ; q)_{m}}{(a q ; q)_{l m}(q ; q)_{m}} t^{m}=\sum_{m=0}^{\infty}\left(a q^{l m+1} ; q\right)_{m} \frac{(b q ; q)_{m}}{(q ; q)_{m}} t^{m}
$$

Replacing $t$ by $a q^{l m}$ in Lemma 2.3(1), we claim that

$$
\begin{aligned}
& \sum_{m=0}^{\infty} \frac{(b q ; q)_{m}}{(q ; q)_{m}} t^{m} \sum_{k=0}^{m}\left[\begin{array}{c}
m \\
k
\end{array}\right]_{q}\left(-a q^{l m}\right)^{k} q^{\frac{k^{2}+k}{2}} \\
& =\sum_{k=0}^{\infty}(-a)^{k} q^{\frac{k^{2}+k}{2}} \sum_{m=k}^{\infty}\left[\begin{array}{c}
m \\
k
\end{array}\right]_{q} t^{m} q^{k l m} \frac{(b q ; q)_{m}}{(q ; q)_{m}} \\
& =\sum_{k=0}^{\infty}(-a)^{k} q^{\frac{k^{2}+k}{2}} \sum_{n=0}^{\infty}\left[\begin{array}{c}
n+k \\
k
\end{array}\right]_{q} t^{n+k} q^{k l(n+k)} \frac{(b q ; q)_{n+k}}{(q ; q)_{n+k}} \\
& =\sum_{k=0}^{\infty}(-a)^{k} q^{\frac{k^{2}+k}{2}} \sum_{n=0}^{\infty} \frac{(q ; q)_{n+k}}{(q ; q)_{k}(q ; q)_{n}} t^{n+k} q^{k l(n+k)} \frac{(b q ; q)_{n+k}}{(q ; q)_{n+k}} \\
& =\sum_{k=0}^{\infty}(-a t)^{k} q^{\frac{(2 l+1) k^{2}+k}{2}} \frac{(b q ; q)_{k}}{(q ; q)_{k}} \sum_{n=0}^{\infty} \frac{\left(b q^{k+1} ; q\right)_{n}}{(q ; q)_{n}} t^{n} q^{k l n} .
\end{aligned}
$$


By substituting $b q^{k}$ and $t q^{k l}$ for $a$ and $t$, respectively, in Lemma 2.3(2), we derive

$$
\sum_{m=0}^{\infty} \frac{(a q ; q)_{(l+1) m}(b q ; q)_{m}}{(a q ; q)_{l m}(q ; q)_{m}} t^{m}=\sum_{k=0}^{\infty} \frac{(b q ; q)_{k}}{(q ; q)_{k}}(-a t)^{k} q^{\frac{(2 l+1) k^{2}+k}{2}} \frac{\left(b t q^{(l+1) k+1} ; q\right)_{\infty}}{\left(t q^{l k} ; q\right)_{\infty}}
$$

Since $(a ; q)_{n}=\frac{(a ; q)_{\infty}}{\left(a q^{n} ; q\right)_{\infty}}$, it follows that the last can be written as

$$
\frac{(b t q ; q)_{\infty}}{(t ; q)_{\infty}} \sum_{k=0}^{\infty} \frac{(b q ; q)_{k}(t ; q)_{l k}}{(q ; q)_{k}(b t q ; q)_{(l+1) k}}(-a t)^{k} q^{\frac{(2 l+1) k^{2}+k}{2}}
$$

Thus, we deduce the identity as desired.

Next, we present alternative proofs of the following results of Kim [6] as an application of Theorem 2.2.

Corollary 2.4 (1)

$$
\sum_{k=0}^{n-1}[k]_{q} t^{k}=\frac{1}{1-q} \sum_{k=0}^{n-1}\left(1-q^{k}\right) t^{k}=\frac{1}{1-q}\left(\frac{1-t^{n}}{1-t}-\frac{1-t^{n} q^{n}}{1-t q}\right) .
$$

(2)

$$
T_{1, q}=\sum_{k=0}^{n-1}[k]_{q} q^{k}=q\left[\begin{array}{l}
n \\
2
\end{array}\right]_{q}=\frac{1}{2}\left([n]_{q}^{2}-\frac{[2 n]_{q}}{[2]_{q}}\right)
$$

Note that it is exactly the same as (1.1).

(3)

$$
\sum_{k=1}^{n}[k]_{q} q^{k-1}=\sum_{k=1}^{n}[k]_{q} q^{2 n-2 k}=\sum_{k=1}^{n}[k]_{q} \frac{1+q^{k}}{1+q} q^{n-k}=\left[\begin{array}{c}
n+1 \\
2
\end{array}\right]_{q}
$$

These are the q-analogues of $\sum_{k=1}^{n} k=\left(\begin{array}{c}n+1 \\ 2\end{array}\right)$.

Proof (1) Replacing both $b$ and $l$ by 0 in Theorem 2.2, we see that

$$
\sum_{k=0}^{\infty} \frac{(a q ; q)_{k}}{(q ; q)_{k}} t^{k}=\frac{1}{(t ; q)_{\infty}} \sum_{k=0}^{\infty} \frac{1}{(q ; q)_{k}}(-a t)^{k} q^{\frac{k^{2}+k}{2}}
$$

After substituting at for $a$ in Lemma 2.3(3), if we apply it to the above, we get

$$
\sum_{k=0}^{\infty} \frac{(a q ; q)_{k}}{(q ; q)_{k}} t^{k}=\frac{(a t q ; q)_{\infty}}{(t ; q)_{\infty}}
$$

Putting $a=q$ in the above, we get

$$
\sum_{k=0}^{\infty} \frac{\left(q^{2} ; q\right)_{k}}{(q ; q)_{k}} t^{k}=\frac{1}{(1-t)(1-t q)}
$$


However, by using the notation defined in Section 1, the left-hand side of the above can be written as

$$
\sum_{k=0}^{\infty} \frac{1-q^{k+1}}{1-q} t^{k}=\sum_{k=0}^{\infty}[k+1]_{q} t^{k}
$$

Thus, from the calculations above and the fact that $[0]_{q}=1$, we derive

$$
\begin{aligned}
\sum_{k=0}^{\infty}[k]_{q} t^{k} & =\sum_{k=0}^{\infty}[k+1]_{q} t^{k+1}=\frac{t}{(1-t)(1-t q)} \\
& =\frac{1}{1-q}\left(\frac{1}{1-t}-\frac{1}{1-t q}\right)=\frac{1}{1-q}\left(\sum_{k=0}^{\infty}\left(1-q^{k}\right) t^{k}\right) .
\end{aligned}
$$

By considering the exponent of $t$, we conclude that

$$
\sum_{k=0}^{n-1}[k]_{q} t^{k}=\frac{1}{1-q} \sum_{k=0}^{n-1}\left(1-q^{k}\right) t^{k}=\frac{1}{1-q}\left(\frac{1-t^{n}}{1-t}-\frac{1-t^{n} q^{n}}{1-t q}\right) .
$$

(2) If we put $t=q$ in (1), we have the first equality

$$
\begin{aligned}
T_{1, q} & =\sum_{k=0}^{n-1}[k]_{q} q^{k}=\frac{1}{1-q}\left(\frac{1-q^{n}}{1-q}-\frac{1-q^{2 n}}{1-q^{2}}\right) \\
& =\frac{q\left(1-q^{n-1}\right)\left(1-q^{n}\right)}{(1-q)\left(1-q^{2}\right)}=q\left[\begin{array}{l}
n \\
2
\end{array}\right]_{q} .
\end{aligned}
$$

On the other hand, a direct calculation gives

$$
\frac{1}{1-q}\left(\frac{1-q^{n}}{1-q}-\frac{1-q^{2 n}}{1-q^{2}}\right)=[\infty]_{q}\left([n]_{q}-\frac{[2 n]_{q}}{[2]_{q}}\right)=\frac{1}{2}\left([n]_{q}^{2}-\frac{[2 n]_{q}}{[2]_{q}}\right)
$$

Therefore, we establish the claim.

(3) It follows from (2) that

$$
\sum_{k=1}^{n}[k]_{q} q^{k-1}=\left[\begin{array}{c}
n+1 \\
2
\end{array}\right]_{q}
$$

Moreover, if we replace $n-1$ by $n$ in (1) and multiply both sides by $q^{2 n}$, we obtain

$$
q^{2 n} \sum_{k=1}^{n}[k]_{q} t^{k}=\frac{q^{2 n}}{1-q}\left([n+1]_{t}-[n+1]_{t q}\right) .
$$

Observe that the identity above with $t=q^{-2}$ turns out to be a Warnaar's identity [13]:

$$
\sum_{k=1}^{n}[k]_{q} q^{2 n-2 k}=\left[\begin{array}{c}
n+1 \\
2
\end{array}\right]_{q}
$$


Finally, on the basis of geometric series, we get

$$
q^{n} \sum_{k=0}^{n} \frac{1-q^{2 k}}{1-q^{2}} t^{k}=\frac{q^{n}}{1-q^{2}}\left(\frac{1-t^{n+1}}{1-t}-\frac{1-\left(t q^{2}\right)^{n+1}}{1-t q^{2}}\right) .
$$

When $t=q^{-1}$ in the above, we have

$$
\begin{aligned}
\sum_{k=0}^{n} \frac{1-q^{2 k}}{1-q^{2}} q^{n-k} & =\frac{q^{n}}{1-q^{2}}\left(\frac{1-q^{-n-1}}{1-q^{-1}}-\frac{1-q^{n+1}}{1-q}\right) \\
& =\frac{\left(1-q^{n}\right)\left(1-q^{n+1}\right)}{(1-q)\left(1-q^{2}\right)}=\left[\begin{array}{c}
n+1 \\
2
\end{array}\right]_{q} .
\end{aligned}
$$

Note that this formula was also derived by several mathematicians such as Schlosser [17] and Warnaar $[13]$. Since $[0]_{q}=0$,

$$
\sum_{k=1}^{n} \frac{1+q^{k}}{1+q}[k]_{q} q^{n-k}=\sum_{k=0}^{n} \frac{\left(1-q^{k}\right)\left(1+q^{k}\right)}{(1-q)(1+q)} q^{n-k}=\left[\begin{array}{c}
n+1 \\
2
\end{array}\right]_{q} .
$$

Thus, by combining (2.3), (2.4), and (2.5), we reach the conclusion.

\section{$3 q$-Consecutive and $q$-analogue of Eulerian numbers $E_{1, q}$ and $E_{2, q}$}

We have studied the infinite sum with linear coefficients for $q$-numbers in the previous section. In this section, we consider the sum with quadratic coefficients, i.e., the following equation.

\section{Lemma 3.1}

$$
\begin{aligned}
\sum_{k=0}^{n-1}[k]_{q}^{2} t^{k} & =\frac{1}{(1-q)^{2}} \sum_{k=0}^{n-1}\left(1-2 q^{k}+q^{2 k}\right) t^{k} \\
& =\frac{1}{(1-q)^{2}}\left(\frac{1-t^{n}}{1-t}-2 \frac{1-t^{n} q^{n}}{1-t q}+\frac{1-t^{n} q^{2 n}}{1-t q^{2}}\right)
\end{aligned}
$$

Theorem 3.2 As a finite sum for $q$, we get

$$
\begin{aligned}
\sum_{k=0}^{n-1}[k]_{q}^{2} q^{k+1} & =[\infty]_{q}\left([\infty]_{q}-[1]_{q}\right)\left([n]_{q}-2 \frac{[2 n]_{q}}{[2]_{q}}+\frac{[3 n]_{q}}{[3]_{q}}\right) \\
& =\frac{1}{3}\left([n]_{q}^{3}-\frac{[3 n]_{q}}{[3]_{q}}\right)-\frac{1}{2}\left([n]_{q}^{2}-\frac{[2 n]_{q}}{[2]_{q}}\right) .
\end{aligned}
$$

Proof Putting $t=q$ in Lemma 3.1, we derive

$$
\begin{aligned}
\sum_{k=0}^{n-1}[k]_{q}^{2} q^{k} & =\frac{1}{(1-q)^{2}}\left(\frac{1-q^{n}}{1-q}-2 \frac{1-q^{2 n}}{1-q^{2}}+\frac{1-q^{3 n}}{1-q^{3}}\right) \\
& =[\infty]_{q}^{2}\left([n]_{q}-2 \frac{[2 n]_{q}}{[2]_{q}}+\frac{[3 n]_{q}}{[3]_{q}}\right)
\end{aligned}
$$

Since $[\infty]_{q}-[1]_{q}=\frac{1}{1-q}-\frac{1-q}{1-q}=\frac{q}{1-q}$, we get the first equality. 
By routine calculations, we have

$$
\begin{aligned}
& \frac{q}{(1-q)^{2}}\left(\frac{1-q^{n}}{1-q}-2 \frac{1-q^{2 n}}{1-q^{2}}+\frac{1-q^{3 n}}{1-q^{3}}\right) \\
& =\frac{q^{2}\left(1-q^{n-1}\right)\left(1-q^{n}\right)}{(1-q)\left(1-q^{2}\right)\left(1-q^{3}\right)}\left\{q^{2}\left(1-q^{n-1}\right)+\left(1-q^{n}\right)\right\} \\
& =\frac{q^{2}[n-1]_{q}[n]_{q}}{[2]_{q}[3]_{q}}\left(q^{2}[n-1]_{q}+[n]_{q}\right) .
\end{aligned}
$$

Moreover, $\frac{1}{3}\left([n]_{q}^{3}-\frac{[3 n]_{q}}{[3]_{q}}\right)-\frac{1}{2}\left([n]_{q}^{2}-\frac{[2 n]_{q}}{[2]_{q}}\right)$ is also equal to

$$
\frac{q^{2}[n-1]_{q}[n]_{q}}{[2]_{q}[3]_{q}}\left(q^{2}[n-1]_{q}^{2}+[n]_{q}\right)
$$

by the definition of $[n]_{q}$. Therefore, the last equality follows.

Theorem 3.3 Let $S_{l}(t)=\sum_{k=0}^{\infty} \frac{\left(q^{2} ; q\right)_{k}^{l}}{(q ; q)_{k}^{l}} t^{k}$. For $l=2$, we obtain

$$
S_{2, n}(q)=\frac{\left(1-q^{n+\frac{1}{2}}\right)\left(1-q^{n}\right)\left(1-q^{n+1}\right)}{\left(1-q^{\frac{3}{2}}\right)(1-q)\left(1-q^{2}\right)}
$$

The other cases $l>2$ will be studied in greater detail in the next section. This was previously proved by Schlosser [17] by using Bailey's terminating very-well-poised balanced ${ }_{10} \phi_{9}$ transformation.

Proof By definition in Section 1, we see that

$$
\begin{aligned}
S_{2, n}(q) & =\sum_{k=1}^{n} \frac{1-q^{2 k}}{1-q}\left(\frac{1-q^{k}}{1-q}\right) q^{\frac{3 n-3 k}{2}} \\
& =\sum_{k=1}^{n} \frac{1+q^{k}}{1+q}\left(\frac{1-q^{k}}{1-q}\right)^{2} q^{\frac{3 n-3 k}{2}} \\
& =\frac{q^{\frac{3}{2} n}}{1+q}\left(\sum_{k=1}^{n}[k]_{q}^{2} q^{-\frac{3}{2} k}+\sum_{k=1}^{n}[k]_{q}^{2} q^{-\frac{1}{2} k}\right) .
\end{aligned}
$$

From Lemma 3.1, we know that

$$
\sum_{k=0}^{n}[k]_{q}^{2} t^{k}=\frac{1}{(1-q)^{2}}\left(\frac{1-t^{n+1}}{1-t}-2 \frac{1-t^{n+1} q^{n+1}}{1-t q}+\frac{1-t^{n+1} q^{2 n+2}}{1-t q^{2}}\right)
$$

Then, the sum of formulas after setting $t=q^{-\frac{3}{2}}$ and $t=q^{-\frac{1}{2}}$ shows that our corollary is true.

Carlitz [18] constructed a $q$-analogue of Eulerian numbers. On the other hand, Kim considered the following functions [19]:

$$
H_{q}(t)=[2]_{q} e^{\frac{t}{1-q}} \sum_{j=0}^{\infty} \frac{(-1)^{j}}{1+q^{j+1}}\left(\frac{1}{1-q}\right)^{j} \frac{t^{j}}{j !}=\sum_{k=0}^{\infty} E_{k, q} \frac{t^{k}}{k !}
$$


and

$$
H_{q}(x, t)=[2]_{q} e^{\frac{t}{1-q}} \sum_{j=0}^{\infty} \frac{(-1)^{j} q^{j x}}{1+q^{j+1}}\left(\frac{1}{1-q}\right)^{j} \frac{t^{j}}{j !}=\sum_{k=0}^{\infty} E_{k, q}(x) \frac{t^{k}}{k !} .
$$

For $m, n \in \mathbb{N}$, he showed that $[19$, Proposition 2]

$$
\sum_{k=0}^{n-1}(-1)^{k}[k]_{q}^{l} q^{k}=\frac{1}{[2]_{q}}\left((-1)^{n+1} q^{n} E_{l, q}(n)+E_{l, q}\right) .
$$

A similar result is in [8, Lemma 2.1]. Thus, we get the results for $l=1$ and 2 as follows.

Theorem 3.4 (1)

$$
(-1)^{n+1} q^{n} E_{1, q}(n)+E_{1, q}= \begin{cases}{[n]_{q}-[2]_{q}^{2} \frac{[2 n]_{q}}{[4]_{q}}} & \text { if } n \text { is even }, \\ {[\infty]_{q}\left(\frac{[2 n]_{q}}{[n]_{q}}-\frac{[2]_{q}^{2}[4 n]_{q}}{[4]_{q}[2 n]_{q}}\right)} & \text { otherwise. }\end{cases}
$$

(2)

$$
\begin{aligned}
& (-1)^{n+1} q^{n} E_{2, q}(n)+E_{2, q} \\
& \quad= \begin{cases}{[\infty]_{q}\left([n]_{q}-2 \frac{[2]_{q}^{2}[2 n]_{q}}{[4]_{q}}+\frac{[2]_{q}[3]_{q}[3 n]_{q}}{[6]_{q}}\right)} & \text { if } n \text { is even, } \\
{[\infty]_{q}^{2}\left(\frac{[2 n]_{q}}{[n]_{q}}-2 \frac{[2]_{q}^{2}[4 n]_{q}}{[4]_{q}[2 n]_{q}}+\frac{[2]_{q}[3]_{q}[6 n]_{q}}{[6]_{q}[3 n]_{q}}\right)} & \text { otherwise. }\end{cases}
\end{aligned}
$$

Proof Replacing $t$ by $-t$ in Corollary 2.4(1), we see that

$$
\sum_{k=0}^{n-1}(-1)^{k}[k]_{q} t^{k}=\frac{1}{1-q}\left(\frac{1-(-t)^{n}}{1+t}-\frac{1-(-t q)^{n}}{1+t q}\right) .
$$

If we let $t=q$, it becomes

$$
\sum_{k=0}^{n-1}(-1)^{k}[k]_{q} q^{k}=\frac{1}{1-q}\left(\frac{1-(-q)^{n}}{1+q}-\frac{1-(-1)^{n} q^{2 n}}{1+q^{2}}\right)
$$

Therefore,

$$
\sum_{k=0}^{n-1}(-1)^{k}[k]_{q} q^{k}= \begin{cases}\frac{[n]_{q}}{[2]_{q}}-\frac{[2 n]_{q}[2]_{q}}{[4]_{q}} & \text { if } n \text { is even, } \\ {[\infty]_{q}\left(\frac{[2 n]_{q}}{[2]_{q}[n]_{q}}-\frac{[2]_{q}[4 n]_{q}}{[4]_{q}[2 n]_{q}}\right)} & \text { otherwise. }\end{cases}
$$

Comparing this with (3.1), we can prove (1).

As for (2), if we substitute $-q$ with $t$ in Lemma 3.1, it turns out that

$$
\sum_{k=0}^{n-1}(-1)^{k}[k]_{q}^{2} q^{k}=\frac{1}{(1-q)^{2}}\left(\frac{1-(-q)^{n}}{1+q}-2 \frac{1-\left(-q^{2}\right)^{n}}{1+q^{2}}+\frac{1-\left(-q^{3}\right)^{n}}{1+q^{3}}\right)
$$


For an even integer $n$, the above becomes

$$
\begin{aligned}
& \frac{1}{1-q}\left(\frac{1-q^{n}}{1-q^{2}}-2 \frac{\left(1-q^{2 n}\right)\left(1-q^{2}\right)}{(1-q)\left(1-q^{4}\right)}+\frac{\left(1-q f o^{3 n}\right)\left(1-q^{3}\right)}{(1-q)\left(1-q^{6}\right)}\right) \\
& \quad=[\infty]_{q}\left(\frac{[n]_{q}}{[2]_{q}}-2 \frac{[2 n]_{q}[2]_{q}}{[4]_{q}}+\frac{[3 n]_{q}[3]_{q}}{[6]_{q}}\right) .
\end{aligned}
$$

Similarly, for an odd integer $n$, we get the result

$$
[\infty]_{q}^{2}\left(\frac{[2 n]_{q}}{[2]_{q}[n]_{q}}-2 \frac{[2]_{q}[4 n]_{q}}{[4]_{q}[2 n]_{q}}+\frac{[3]_{q}[6 n]_{q}}{[6]_{q}[3 n]_{q}}\right)
$$

as desired.

Remark 3.5 In the proof above, as in the case of (3.2), we can obtain an equation by plugging $-q$ into $t$ :

$$
\sum_{k=0}^{n-1}[k]_{q} q^{k}=\frac{1}{1-q}\left(\frac{1-q^{n}}{1-q}-\frac{1-q^{2 n}}{1-q^{2}}\right) .
$$

Further, we have $2 \sum_{k=0}^{n-1}[2 k]_{q} q^{2 k}$ by adding (3.2) and (3.3). Indeed,

$$
\begin{aligned}
& \sum_{k=0}^{2 n-1}(-1)^{k}[k]_{q} q^{k}+\sum_{k=0}^{2 n-1}[k]_{q} q^{k} \\
& \quad=\frac{1}{1-q}\left(\frac{1-q^{2 n}}{1+q}-\frac{1-q^{4 n}}{1+q^{2}}\right)+\frac{1}{1-q}\left(\frac{1-q^{2 n}}{1-q}-\frac{1-q^{4 n}}{1-q^{2}}\right) \\
& \quad=\frac{2}{1-q}\left(\frac{1-q^{2 n}}{1-q^{2}}-\frac{1-q^{4 n}}{1-q^{4}}\right) .
\end{aligned}
$$

Note that this can be written as $2[\infty]_{q}\left(\frac{[2 n]_{q}}{[2]_{q}}-\frac{[4 n]_{q}}{[4]_{q}}\right)$ in terms of $q$-number notation. Alternatively, it may be factorized and expressed as

$$
\frac{2 q^{2}\left(1-q^{2 n-2}\right)\left(1-q^{2 n}\right)}{(1-q)\left(1-q^{4}\right)}=\frac{2 q^{2}[2 n-2]_{q}[2 n]_{q}}{[4]_{q}},
$$

from which we derive

$$
\sum_{k=0}^{n-1}[2 k]_{q} q^{2 k}=[\infty]_{q}\left(\frac{[2 n]_{q}}{[2]_{q}}-\frac{[4 n]_{q}}{[4]_{q}}\right)=\frac{q^{2}[2 n-2]_{q}[2 n]_{q}}{[4]_{q}}
$$

Remark 3.6 In [15, Theorem 1], Kim derived a summation formula for $E_{m, q}$,

$$
E_{m, q}=[2]_{q}\left(\frac{1}{1-q}\right)^{m} \sum_{k=0}^{m}\left(\begin{array}{c}
m \\
k
\end{array}\right)(-1)^{k} \frac{1}{1+q^{k+1}} .
$$

We also see one for $E_{k, q}(x)$,

$$
E_{k, q}(x)=[2]_{q} \sum_{m=1}^{\infty}(-1)^{n} q^{n}[n+x]_{q}^{k}
$$


for any positive integer $k$ from [20, Proposition 1]. The proof of Theorem 3.4 is obtained without the help of the summations above.

\section{Difference equation and $q$-consecutive integer}

As mentioned in Theorem 3.3, in this section, we study $\sum_{k=0}^{\infty} \frac{\left(q^{2} ; q\right)_{k}^{l}}{(q ; q)_{k}^{l}} t^{k}$ for more general cases $l$ and its similar sum with $q$-binomial coefficients. In addition, we show the relations between these and twisted $q$-Bernoulli numbers. To this end, we need the following lemma.

Lemma 4.1 Given a sequence $A_{k}(k \geq 0)$ for which $g(t)=\sum_{k=0}^{\infty} A_{k} t^{k}$ converges,

$$
\sum_{k=0}^{\infty} \frac{(a q ; q)_{k}}{(b q ; q)_{k}} A_{k} t^{k}=\frac{(a q ; q)_{\infty}}{(b q ; q)_{\infty}} \sum_{k=0}^{\infty} \frac{(b / a ; q)_{k}}{(q ; q)_{k}} a^{k} q^{k} g\left(t q^{k}\right) .
$$

Proof See Section 20, [14].

Using this lemma, we generalize the identities considered in the previous two sections.

Proposition 4.2 For a positive integer $l$, we have the identities

$$
\begin{aligned}
\sum_{k=0}^{n-1}[k]_{q}^{l} q^{k} & =\frac{q}{(1-q)^{l}}\left(\frac{1-q^{n-1}}{1-q}-l q \frac{1-q^{2(n-1)}}{1-q^{2}}+\cdots+(-1)^{l} q^{l} \frac{1-q^{(l+1)(n-1)}}{1-q^{l+1}}\right) \\
& =\frac{q}{(1-q)^{l}} \sum_{k=0}^{l}\left(\begin{array}{l}
l \\
k
\end{array}\right) \frac{1-q^{(k+1)(n-1)}}{1-q^{k+1}}(-q)^{k} .
\end{aligned}
$$

Ifl is 1 (respectively, 2), this would be the result of Corollary 2.4(1) (respectively, Lemma 3.1).

Proof Let $S_{l}(t)$ be a series defined by $\sum_{k=0}^{\infty} \frac{\left(q^{2} ; q\right)_{k}^{l}}{(q ; q)_{k}^{l}} t^{k}$ for a nonnegative integer $l$. By setting $a=q, b=1, A_{k}=\frac{\left(q^{2} ; q\right)_{k}^{l}}{(q ; q)_{k}^{l}}$, and $g(t)=S_{l}(t)$ in Lemma 4.1, we derive the following recursive formula:

$$
\begin{aligned}
S_{l+1}(t) & =\sum_{k=0}^{\infty} \frac{\left(q^{2} ; q\right)_{k}}{(q ; q)_{k}} \frac{\left(q^{2} ; q\right)_{k}^{l}}{(q ; q)_{k}^{l}} t^{k}=\frac{\left(q^{2} ; q\right)_{\infty}}{(q ; q)_{\infty}} \sum_{k=0}^{\infty} \frac{(1 / q ; q)_{k}}{(q ; q)_{k}} q^{2 k} S_{l}\left(t q^{k}\right) \\
& =\frac{1}{1-q}\left(S_{l}(t)+\frac{1-\frac{1}{q}}{1-q} q^{2} S_{l}(t q)\right)=\frac{1}{1-q}\left(S_{l}(t)-q S_{l}(t q)\right) .
\end{aligned}
$$

Multiplying both sides by $t$, we get

$$
\begin{aligned}
& t S_{1}(t)=\frac{t}{1-q}\left(S_{0}(t)-q S_{0}(t q)\right) \\
& t S_{2}(t)=\frac{t}{1-q}\left(S_{1}(t)-q S_{1}(t q)\right)=\frac{t}{(1-q)^{2}}\left(S_{0}(t)-2 q S_{0}(t q)+q^{2} S_{0}\left(t q^{2}\right)\right) .
\end{aligned}
$$

Further, by induction,

$$
t S_{l}(t)=\frac{t}{(1-q)^{l}} \sum_{k=0}^{l}\left(\begin{array}{l}
l \\
k
\end{array}\right) S_{0}\left(t q^{k}\right)(-q)^{k} .
$$


Considering $t S_{0}(t)=t \sum_{k=0}^{\infty} t^{k}=\frac{t}{1-t}$, we are able to rewrite the above as

$$
\begin{aligned}
& \sum_{k=0}^{\infty}[k]_{q} t^{k}=\frac{t}{1-q}\left(\frac{1}{1-t}-\frac{q}{1-t q}\right) \\
& \sum_{k=0}^{\infty}[k]_{q}^{2} t^{k}=\frac{t}{(1-q)^{2}}\left(\frac{1}{1-t}-\frac{2 q}{1-t q}+\frac{q^{2}}{1-t q^{2}}\right), \\
& \ldots, \\
& \sum_{k=0}^{\infty}[k]_{q}^{l} t^{k}=\frac{t}{(1-q)^{l}}\left(\frac{1}{1-t}-\frac{l q}{1-t q}+\cdots+\frac{(-q)^{l}}{1-t q^{l}}\right)=\frac{t}{(1-q)^{l}} \sum_{k=0}^{l}\left(\begin{array}{l}
l \\
k
\end{array}\right) \frac{(-q)^{k}}{1-t q^{k}}
\end{aligned}
$$

If we take a finite sum from the above, we get

$$
\begin{aligned}
\sum_{k=0}^{n-1}[k]_{q}^{l} t^{k} & =\frac{t}{(1-q)^{l}}\left(\sum_{k=0}^{n-2} t^{k}-l q \sum_{k=0}^{n-2} t^{k} q^{k}+\cdots+(-q)^{l} \sum_{k=0}^{n-2} t^{k} q^{l k}\right) \\
& =\frac{t}{(1-q)^{l}}\left(\frac{1-t^{n-1}}{1-t}-l q \frac{1-t^{n-1} q^{n-1}}{1-t q}+\cdots+(-q)^{l} \frac{1-t^{n-1} q^{l(n-1)}}{1-t q^{l}}\right) \\
& =\frac{t}{(1-q)^{l}} \sum_{k=0}^{l}\left(\begin{array}{l}
l \\
k
\end{array}\right) \frac{1-\left(t q^{k}\right)^{n-1}}{1-t q^{k}}(-q)^{k} .
\end{aligned}
$$

Therefore, if we let $t=q$, we are done, which amounts to recovering Corollary 2.4, Lemma 3.1, and Theorem 3.2.

Remark 4.3 In Section 1, we mentioned Kim's relation about $q$-Bernoulli polynomials and $q$-consecutive integers, from which we obtain some identities for $\int_{0}^{n} \beta_{l, q} d[x]_{q}$, namely

$$
\begin{aligned}
\int_{0}^{n} \beta_{l, q} d[x]_{q} & =\frac{1}{l+1}\left(\beta_{l+1, q}(n)-\beta_{l+1, q}\right)=T_{l, q}(n) \\
& =\sum_{k=0}^{n-1}[k]_{q}^{l} q^{k}=\frac{1}{(1-q)^{l}} \sum_{k=0}^{l}(-1)^{k}\left(\begin{array}{l}
l \\
k
\end{array}\right) \frac{1-q^{(k+1)(n-1)}}{1-q^{k+1}} q^{k+1} \\
& =[\infty]_{q}^{l} \sum_{k=0}^{l}\left(\begin{array}{l}
l \\
k
\end{array}\right)(-1)^{k}[n-1]_{q^{k+1}} q^{k+1} .
\end{aligned}
$$

Next, we would like to consider the sum $T_{l, t}$ from (4.2) when $l=1$,

$$
T_{1, t}=\sum_{k=0}^{\infty}[k]_{q} t^{k-1}=\frac{1}{(1-t)(1-t q)} .
$$

By the same argument as that in the proof of Proposition 4.2, we have more general identities for $T_{l, t}$ :

$$
T_{2, t}=\sum_{k=0}^{\infty}[k]_{q}^{2} t^{k-1}=\frac{1+t q}{(1-t)(1-t q)\left(1-t q^{2}\right)}=: g(t)
$$




$$
\begin{aligned}
T_{3, t} & =\sum_{k=0}^{\infty}[k]_{q}^{3} t^{k-1}=\frac{1}{1-q}\{g(t)-q g(t q)\} \\
& =\frac{1}{1-q}\left(\frac{1+t q}{(1-t)(1-t q)\left(1-t q^{2}\right)}-\frac{q\left(1+t q^{2}\right)}{(1-t q)\left(1-t q^{2}\right)\left(1-t q^{3}\right)}\right) \\
& =\frac{1+2 t q+2 t q^{2}+t^{2} q^{3}}{(1-t)(1-t q)\left(1-t q^{2}\right)\left(1-t q^{3}\right)} \quad \text { and } \\
T_{4, t} & =\sum_{k=0}^{\infty}[k]_{q}^{4} t^{k-1}=\frac{\left(1+t q^{2}\right)\left(1+3 t q+4 t q^{2}+3 t q^{3}+t^{2} q^{4}\right)}{(1-t)(1-t q)\left(1-t q^{2}\right)\left(1-t q^{3}\right)\left(1-t q^{4}\right)}
\end{aligned}
$$

All the denominators on the right-hand side are factorized as $l+1$ terms. However, the numerators are somewhat complex. Therefore, we recursively define a sequence $C_{l}(t ; q)$ with $l \geq 1$ as follows:

$$
\begin{aligned}
& C_{0}(t ; q)=1 \\
& C_{l}(t ; q)=\frac{1}{1-q}\left\{\left(1-t q^{l}\right) C_{l-1}(t ; q)-q(1-t) C_{l-1}(t q ; q)\right\} .
\end{aligned}
$$

Then, we get the following theorem.

Theorem 4.4 (1) The infinite sum $T_{l, t}$ is expressed as a quotient of $C_{l}(t ; q)$ by $l+1$ products, precisely speaking,

$$
T_{l, t}=\sum_{k=0}^{\infty}[k]_{q}^{l} t^{k-1}=\frac{C_{l}(t ; q)}{(t ; q)_{l+1}} .
$$

(2) Since $C_{l}(q ; q)=C_{l-1}(q ; q)[l+1]_{q}-q C_{l-1}\left(q^{2} ; q\right)$, we have

$$
\sum_{k=0}^{\infty}[k]_{q}^{l} q^{k-1}=\frac{C_{l}(q ; q)}{(q ; q)_{l+1}}
$$

Replacing $t$ by $\frac{1}{t}$ in Theorem 4.4(1), we can deduce one of Simsek's relations [9, Proposition 3.1].

Theorem 4.5 The generating function (complex cases) of twisted q-Bernoulli numbers is given by

$$
f_{q, \omega}(t)=\sum_{k=0}^{\infty} B_{k, \omega}^{*}(q) \frac{t^{k}}{k !},
$$

where $\omega$ is the rth root of unity and

$$
B_{l, \omega}^{*}(q)= \begin{cases}0 & \text { if } l=0, \\ \frac{\omega q}{1-\omega q} & \text { if } l=1, \\ \frac{l \omega^{l-1} q^{\frac{l(l-1)}{2}}}{(\omega q ; q)_{l}} C_{l-1}\left(\omega^{-1} q^{-l} ; q\right) & \text { if } l \geq 2\end{cases}
$$


Proof If we recall (S1) from Section 1, we get

$$
\begin{aligned}
B_{0, \omega}^{*}(q) & =0 \\
B_{1, \omega}^{*}(q) & =-\sum_{k=0}^{\infty} \omega^{-k} q^{-k}=\frac{\omega q}{1-\omega q} \\
B_{2, \omega}^{*}(q) & =2 \sum_{k=0}^{\infty} \omega^{-k} q^{-2 k}[k]_{q}=\frac{2}{w q^{2}} \sum_{k=0}^{\infty}[k]_{q} w^{-k+1} q^{-2 k+2} \\
& =\frac{2}{\omega q^{2}} \sum_{k=1}^{\infty}[k]_{q} \omega^{-k+1} q^{-2 k+2}=\frac{2}{\omega q^{2}} \sum_{k=0}^{\infty} \frac{\left(q^{2} ; q\right)_{k}}{(q ; q)_{k}} \omega^{-k} q^{-2 k} \\
& =\frac{2}{\omega q^{2}} F\left(q, 1 ; \omega^{-1} q^{-2}: q\right) .
\end{aligned}
$$

Since we know from $[14,(6.2)]$ that

$$
F(a, 1 ; t: q)=\frac{(a t q: q)_{\infty}}{(t: q)_{\infty}}
$$

by setting $q$ and $t$ to be $a$ and $\omega^{-1} q^{-2}$, respectively, we obtain

$$
B_{2, \omega}^{*}(q)=\frac{2 \omega q}{(1-\omega q)\left(1-\omega q^{2}\right)} .
$$

When $l$ is greater than 2 , we get

$$
\begin{aligned}
B_{l, \omega}^{*}(q) & =(-1)^{l} l \sum_{k=0}^{\infty} \omega^{-k} q^{-l k}[k]_{q}^{l-1}=\frac{(-1)^{l} l}{\omega q^{l}} T_{l-1, \omega^{-1} q^{-l}} \\
& =\frac{(-1)^{l} l}{\omega q^{l}} \frac{C_{l-1}\left(\omega^{-1} q^{-l} ; q\right)}{\left(\omega^{-1} q^{-l} ; q\right)_{l}}=\frac{l \omega^{l-1} q^{\frac{l(l-1)}{2}}}{(\omega q ; q)_{l}} C_{l-1}\left(\omega^{-1} q^{-l} ; q\right) .
\end{aligned}
$$

By (S2) and Theorem 4.5, we get a corollary.

Corollary 4.6 If $l$ is an integer greater than 1, we have

$$
\zeta_{\omega, q}(1-l)=(-1)^{l+1} \frac{\omega^{l-1} q^{\frac{l(l-1)}{2}}}{(\omega q ; q)_{l}} C_{l-1}\left(\omega^{-1} q^{-l} ; q\right) .
$$

Theorem 4.7 For any integer $x$,

$$
B_{l, \omega}^{*}(x, q)= \begin{cases}\frac{-\omega q}{1-\omega q} & \text { if } l=1, \\
\frac{2\left(1-\omega q-q^{x}+\omega q^{x+2}\right)}{\omega q^{2}(1-q)(1-\omega q)\left(1-\omega q^{2}\right)} & \text { if } l=2, \\
\omega^{x} q^{l x}\left(-\frac{l \omega^{l} q^{\frac{l(l-1)}{2}}}{(\omega q ; q)} C_{l}\left(\omega^{-1} q^{-l} ; q\right)\right. & \\
\left.\quad+\frac{(-1)^{l} l}{(1-q)^{l-1}} \sum_{k=0}^{l-1}\left(\begin{array}{c}
l-1 \\
k
\end{array}\right)[x]_{\omega q^{l-k}} \omega^{-x} q^{-l x}\left(-q^{x}\right)^{k}\right) & \text { if } l>2 .\end{cases}
$$


Proof It follows from (3.6) in [9] that

$$
\begin{aligned}
B_{l, \omega}^{*}(x, q) & =(-1)^{l+1} l \sum_{k=1}^{\infty} \omega^{-k} q^{-k l}[k+x]_{q}^{l-1} \\
& =\omega^{x} q^{l x}\left((-1)^{l+1} l \sum_{k=1}^{\infty} \omega^{-k} q^{-k l}[k]_{q}^{l-1}-(-1)^{l+1} l \sum_{k=1}^{x} \omega^{-k} q^{-k l}[k]_{q}^{l-1}\right)
\end{aligned}
$$

with $x \in \mathbb{N}$. By direct calculation, we get the identities

$$
\begin{aligned}
& B_{1, \omega}^{*}(x, q)=\sum_{k=1}^{\infty} \omega^{-k} q^{-k}=-\frac{\omega q}{1-\omega q} \\
& B_{2, \omega}^{*}(x, q)=-2 \sum_{k=1}^{\infty} \omega^{-k} q^{-2 k} \frac{1-q^{k+x}}{1-q}=\frac{2\left(1-\omega q-q^{x}+\omega q^{x+2}\right)}{\omega q^{2}(1-q)(1-\omega q)\left(1-\omega q^{2}\right)}
\end{aligned}
$$

and when $l>2$, we derive from Proposition 4.2 and Theorem 4.5 that

$$
B_{l, \omega}^{*}(x, q)=\omega^{x} q^{x}\left(-B_{l, \omega}^{*}(q)+(-1)^{l} l \sum_{k=1}^{x} \frac{1}{\omega^{k} q^{l k}}[k]_{q}^{l-1}\right) \text {. }
$$

Substituting $l-1, x+1$, and $\omega^{-1} q^{-l}$ for $l, n$, and $t$ in (4.3), respectively, we establish the last identities.

As its immediate corollary, we have the following.

\section{Corollary 4.8}

$$
\zeta_{\omega, q}(1-l, x)= \begin{cases}-\frac{\omega q}{1-\omega q} & \text { if } l=1, \\
-\frac{1-\omega q-q^{x}+\omega q^{x+2}}{\omega q^{2}(1-q)(1-\omega q)\left(1-\omega q^{2}\right)} & \text { if } l=2, \\
\omega^{x} q^{l x}\left((-1)^{l} \frac{\omega^{l} q^{\frac{l(l-1)}{2}}}{(\omega q ; q)_{l}} C_{l}\left(\omega^{-1} q^{-l} ; q\right)\right. & \\
-\frac{1}{(1-q)^{l-1}} \sum_{k=0}^{l-1}\left(\begin{array}{c}
l-1 \\
k
\end{array}\right)[x]_{\left.\omega q^{l-k} \omega^{-x} q^{-l x}\left(-q^{x}\right)^{k}\right)} & \text { if } l>2 .\end{cases}
$$

Moreover, we can deduce the following corollary, which is analogous to Theorem 4.4.

\section{Corollary 4.9}

$$
\sum_{k=1}^{\infty}[k]_{q}^{l} \frac{1+q^{k}}{1+q} t^{k-1}=\frac{1}{1+q}\left(\frac{C_{l}(t ; q)}{(t ; q)_{l+1}}+q \frac{C_{l}(t q ; q)}{(t q ; q)_{l+1}}\right) .
$$

Proposition 4.10 For a nonnegative integer $n$,

(1) $\sum_{k=0}^{\infty}\left[\begin{array}{c}k+n \\ n\end{array}\right]_{q} t^{k}=\frac{1}{(t ; q)_{n+1}}$,

and when $n=2$, we get the following by considering the summation from 0 to $n-1$ in the above:

(2) $\sum_{k=0}^{n-1}\left[\begin{array}{c}k+2 \\ 2\end{array}\right]_{q} q^{k}=\left[\begin{array}{c}n+2 \\ 3\end{array}\right]_{q}$ 
Furthermore, we obtain

(3) $\sum_{k=0}^{\infty}\left[\begin{array}{c}k+2 \\ 2\end{array}\right]_{q}^{2} q^{k}=\frac{1+q^{2}+2 q^{3}+q^{4}+q^{6}}{(1-q)\left(1-q^{2}\right)\left(1-q^{3}\right)\left(1-q^{4}\right)\left(1-q^{5}\right)}$.

Proof By Lemma 2.3(2), we get

$$
\sum_{k=0}^{\infty}\left[\begin{array}{c}
k+n \\
n
\end{array}\right]_{q} t^{k}=\sum_{k=0}^{\infty} \frac{\left(q^{n+1} ; q\right)_{k}}{(q ; q)_{k}} t^{k}=\frac{1}{(t ; q)_{n+1}} .
$$

As for the second, we set $n=2$. Then, it follows from Lemma 4.1 that

$$
\sum_{k=0}^{\infty}\left[\begin{array}{c}
k+2 \\
2
\end{array}\right]_{q} t^{k}=\frac{\left(q^{3} ; q\right)_{\infty}}{(q ; q)_{\infty}} \sum_{k=0}^{\infty} \frac{\left(q^{-2} ; q\right)_{k}}{(q ; q)_{k}} q^{3 k} \sum_{l=0}^{\infty} t^{l} q^{l k}
$$

Considering that the exponent of $t$ is less than $n$ only in the above, we have

$$
\begin{aligned}
\sum_{k=0}^{n-1}\left[\begin{array}{c}
k+2 \\
2
\end{array}\right]_{q} t^{k}= & \frac{\left(q^{3} ; q\right)_{\infty}}{(q ; q)_{\infty}} \sum_{k=0}^{\infty} \frac{\left(q^{-2} ; q\right)_{k}}{(q ; q)_{k}} q^{3 k} \sum_{l=0}^{n-1} t^{l} q^{2 l} \\
= & \frac{1}{(1-q)\left(1-q^{2}\right)}\left(\sum_{l=0}^{n-1} t^{l}+\frac{\left(1-q^{-2}\right)}{1-q} q^{3} \sum_{l=0}^{n-1} t^{l} q^{l}\right. \\
& \left.+\frac{\left(1-q^{-2}\right)\left(1-q^{-1}\right)}{(1-q)\left(1-q^{2}\right)} q^{6} \sum_{l=0}^{n-1} t^{l} q^{2 l}\right)
\end{aligned}
$$

Then, putting $t=q$ we get

$$
\begin{aligned}
& \frac{1}{(1-q)\left(1-q^{2}\right)}\left(\frac{1-q^{n}}{1-q}-\frac{q(1+q)\left(1-q^{2 n}\right)}{1-q^{2}}+\frac{q^{3}\left(1-q^{3 n}\right)}{1-q^{3}}\right) \\
& =\frac{\left(1-q^{n}\right)\left(1-q^{n+1}\right)\left(1-q^{n+2}\right)}{(1-q)\left(1-q^{2}\right)\left(1-q^{3}\right)}=\left[\begin{array}{c}
n+2 \\
3
\end{array}\right]_{q} .
\end{aligned}
$$

In order to show (3), let $g(t)=\sum_{k=0}^{\infty} \frac{\left(q^{3} ; q\right)_{k}}{(q ; q)_{k}} t^{k}=\frac{1}{(t ; q)_{3}}$. Then, we derive

$$
\begin{aligned}
\sum_{k=0}^{\infty}\left[\begin{array}{c}
k+2 \\
2
\end{array}\right]_{q}^{2} t^{k} & =\sum_{k=0}^{\infty} \frac{\left(q^{3} ; q\right)_{k}}{(q ; q)_{k}} \frac{\left(q^{3} ; q\right)_{k}}{(q ; q)_{k}} t^{k} \\
& =\frac{\left(q^{3} ; q\right)_{\infty}}{(q ; q)_{\infty}} \sum_{k=0}^{\infty} \frac{\left(q^{-2} ; q\right)_{k}}{(q ; q)_{k}} q^{3 k} g\left(t q^{k}\right) \\
& =\frac{1}{(1-q)\left(1-q^{2}\right)}\left(g(t)+\frac{1-q^{-2}}{1-q} q^{3} g(t q)+\frac{\left(1-q^{-2}\right)\left(1-q^{-1}\right)}{(1-q)\left(1-q^{2}\right)} q^{6} g\left(t q^{2}\right)\right) \\
& =\frac{1}{(1-q)\left(1-q^{2}\right)}\left(\frac{1}{(t ; q)_{3}}-\frac{q(1+q)}{(t q ; q)_{3}}+\frac{q^{3}}{\left(t q^{2} ; q\right)_{3}}\right) \\
& =\frac{1+t q+2 t q^{2}+t q^{3}+t^{2} q^{4}}{(1-t)(1-t q)\left(1-t q^{2}\right)\left(1-t q^{3}\right)\left(1-t q^{4}\right)}
\end{aligned}
$$

Thus, by substituting $q$ for $t$, we conclude (3). 
Proposition 4.11 For a nonnegative integer $l$,

(1) $\sum_{k=0}^{\infty}[k]_{q}^{l} t^{k-1}=C_{l}(t ; q) \sum_{k=0}^{\infty}\left[\begin{array}{c}l+k \\ k\end{array}\right]_{q} t^{k}$,
(2) $\sum_{k=0}^{\infty}[k]_{q}^{l} q^{k-1}=C_{l}(q ; q) \sum_{k=0}^{\infty}\left[\begin{array}{c}l+k \\ k\end{array}\right]_{q} q^{k}$.

Proof We see from (6.22) in [14] that $\sum_{k=0}^{\infty}\left(\begin{array}{c}l+k \\ k\end{array}\right)_{q} t^{k}=\frac{1}{(t ; q)_{l+1}}$. Thus, the proposition follows from Theorem 4.4(2).

Henceforth, we concentrate on $S_{l, n}(t)$ introduced in Section 1.

Theorem 4.12 For a complex number $s$ with $|s|<1$ and positive integers $m$ and $n$,

$$
S_{l, n}(q)=\frac{q^{\frac{(l+1) n}{2}}}{(1+q)(1-q)^{l}} \sum_{m=0}^{l}\left(\begin{array}{c}
l \\
m
\end{array}\right)(-q)^{m}\left(\sum_{k=0}^{n-1} q^{\left(m-\frac{l+1}{2}\right) k}-q \sum_{k=0}^{n-1}\left(q^{\left(m+1-\frac{l+1}{2}\right) k}\right)\right) .
$$

Here, we consider $\left(\begin{array}{l}0 \\ 0\end{array}\right)$ as 1 .

Proof For fixed $n$, we consider $\sum_{k=0}^{n-1}[k+1]_{q}^{l} \frac{1+q^{k+1}}{1+q} t^{k}$, and we denote it by $g_{l}(t)$ so that $g_{0}(t)=$ $\frac{1}{1+q}\left(\sum_{k=0}^{n-1} t^{k}-q \sum_{k=0}^{n-1} t^{k} q^{k}\right)$. By adopting the arguments used in Lemma 4.1, we obtain the following recursion

$$
\begin{aligned}
g_{l+1}(t) & =\sum_{k=0}^{n-1} \frac{1-q^{k+1}}{1-q}[k+1]_{q}^{l} \frac{1+q^{k+1}}{1+q} t^{k} \\
& =\sum_{k=0}^{n} \frac{\left(q^{2} ; q\right)_{k}}{(q ; q)_{k}}[k+1]_{q}^{l} \frac{1+q^{k+1}}{1+q} t^{k} \\
& =\frac{\left(q^{2} ; q\right)_{\infty}}{(q ; q)_{\infty}} \sum_{k=0}^{\infty} \frac{\left(\frac{1}{q} ; q\right)_{k}}{(q ; q)_{k}} q^{2 k} g_{l}\left(t q^{k}\right) \\
& =\frac{1}{1-q}\left(g_{l}(t)-q g_{l}(t q)\right) .
\end{aligned}
$$

Since the above is true for all adjacent integers, we obtain

$$
g_{l}(t)=\frac{1}{(1-q)^{l}} \sum_{k=0}^{l}\left(\begin{array}{l}
l \\
k
\end{array}\right)(-q)^{k} g_{0}\left(t q^{k}\right)
$$

Multiplying both sides by $q^{\frac{(l+1) n}{2}}$ and replacing $t$ by $q^{-\frac{l+1}{2}}$, we complete the proof.

Remark 4.13 In Section 1, we mentioned that the generating function for the rank of partition can be written as $T_{l, t}$ and $C_{l}(t ; q)$ :

$$
\sum_{l=0}^{\infty} \frac{T_{l, \frac{q}{u}}}{C_{l}\left(u^{-1} q ; q\right)} u^{l} q^{l+1}=\sum_{r=-\infty}^{\infty} \sum_{n=1}^{\infty} P_{r}(n) u^{r} q^{n}
$$


By letting $u=-1$ on the right-hand side, we get

$$
\begin{aligned}
\sum_{r=-\infty}^{\infty} \sum_{n=1}^{\infty} P_{r}(n)(-1)^{r} & =\sum_{n=1}^{\infty}\left(\sum_{r: \text { even }} P_{r}(n)-\sum_{r: \text { odd }} P_{r}(n)\right) q^{r} \\
& =\sum_{n=1}^{\infty} \frac{q^{n^{2}}}{(-q ; q)_{n}^{2}} .
\end{aligned}
$$

Further, it gives rise to a third-order mock theta function $f(q)=1+\sum_{n=1}^{\infty} \alpha(n) q^{n}=1+$ $\sum_{n=1}^{\infty} \frac{q^{n^{2}}}{(-q ; q)_{n}^{2}}$, where the explicit formula of $\alpha(n)$ was conjectured by both Andrews [21] and Dragonette [22], and later proved by Bringmann and Ono [23]. Let $N_{e}(n)$ (resp., $\left.N_{o}(n)\right)$ be the infinite sum $\sum_{r \text { : even }} P_{r}(n)$ (resp., $\sum_{r \text { : odd }} P_{r}(n)$ ). Then, we can find the coefficients of the infinite sum $\sum_{n=0}^{\infty} \frac{T_{n,-q}(-1)^{n}}{C_{n}(-q ; q)} q^{n+1}$, because the formula for the partition function $p(n)$ is already known and $\alpha(n)=N_{e}(n)-N_{o}(n)$.

Remark 4.14 In Ramanujan's lost notebook, there are 4 third-order mock theta functions $[24$, p.345]:

$$
\begin{aligned}
& f(q)=\sum_{k=0}^{\infty} \frac{q^{k^{2}}}{(-q ; q)_{k}^{2}}, \\
& \phi(q)=1+\frac{q}{1+q^{2}}+\frac{q^{4}}{\left(1+q^{2}\right)\left(1+q^{4}\right)}+\frac{q^{9}}{\left(1+q^{2}\right)\left(1+q^{4}\right)\left(1+q^{6}\right)}+\cdots, \\
& \psi(q)=\frac{q}{1-q}+\frac{q^{4}}{(1-q)\left(1-q^{3}\right)}+\frac{q^{9}}{(1-q)\left(1-q^{3}\right)\left(1-q^{5}\right)}+\cdots, \\
& \chi(q)=1+\frac{q}{1-q+q^{2}}+\frac{q^{4}}{\left(1-q+q^{2}\right)\left(1-q^{2}+q^{4}\right)}+\cdots
\end{aligned}
$$

By utilizing our notations, we interpret them as follows.

From Theorem 4.4, Proposition 4.10, Proposition 4.11, and the definition of the mock theta functions [14, pp.55-57], we are able to connect $q$-consecutive integers with these mock theta functions, namely

$$
\begin{aligned}
& 1+\sum_{l=0}^{\infty} \frac{T_{l, q}}{C_{l}(q ; q)} q^{l+1}=\frac{1}{(q ; q)_{\infty}}=\sum_{k=0}^{\infty} p(k) q^{k}, \\
& 1+\sum_{l=0}^{\infty} \frac{T_{l,-q}(-1)^{l}}{C_{l}(-q ; q)} q^{l+1}=f(q), \\
& 1+\sum_{l=0}^{\infty} \frac{T_{l,-i q}}{C_{l}(-i q ; q)}(i)^{l} q^{l+1}=\phi(q), \\
& 1+\sum_{l=0}^{\infty} \frac{T_{l,-\omega q}}{C_{l}(-\omega q ; q)}\left(-\omega^{2}\right)^{l} q^{l+1}=\chi(q),
\end{aligned}
$$

with $i=e^{2 \pi i / 4}$ and $\omega=e^{2 \pi i / 3}$. 
Authors' contributions

The authors worked on the results independently. All authors read and approved the final manuscript.

\section{Author details}

${ }^{1}$ National Institute for Mathematical Sciences, Yuseong-daero 1689-gil, Yuseong-gu, Daejeon, 305-811, South Korea. ${ }^{2}$ Department of Mathematical Sciences, Korea Advanced Institute of Science and Technology, 373-1 Guseong-dong, Yuseong-gu, Daejeon, 305-701, South Korea. ${ }^{3}$ School of Mathematics, Korea Institute for Advanced Study (KIAS), 85 Hoegiro, Dongdaemun-gu, Seoul, 130-722, South Korea.

\section{Acknowledgements}

The first author was supported by the National Institute for Mathematical Science (NIMS) grant funded by the Korean government (B21303), the second named author partially was supported by the NRF of Korea grant funded by the MISP (2013042157) and the corresponding author was supported by NRF 2012-0006901.

Received: 31 July 2013 Accepted: 5 September 2013 Published: 07 Nov 2013

\section{References}

1. Hegazi, AS, Mansour, M: A note on q-Bernoulli numbers and polynomials. J. Nonlinear Math. Phys. 13(1), 9-18 (2006)

2. Kim, M-S: On Euler numbers, polynomials and related $p$-adic integrals. J. Number Theory 129, 2166-2179 (2009)

3. Kim, T: Power series and asymptotic series associated with the $q$-analog of the two-variable $p$-adic $L$-function. Russ. J. Math. Phys. 12(2), 186-196 (2005)

4. Kupershmidt, BA: Reflection symmetries of $q$-Bernoulli polynomials. J. Nonlinear Math. Phys. 12, 412-422, suppl. 1 (2005)

5. Kim, T: On explicit formulas of $p$-adic $q$-L-functions. Kyushu J. Math. 48(1), 73-86 (1994)

6. Kim, T: Sums of powers of consecutive $q$-integers. Adv. Stud. Contemp. Math. 9(1), 15-18 (2004)

7. Kim, T: Symmetry of power sum polynomials and multivariate fermionic $p$-adic invariant integral on $\mathbb{Z}_{p}$. Russ. J. Math. Phys. 16(1), 93-96 (2009)

8. Kim, M-S, Lee, J: On sums of products of the extended q-Euler numbers. J. Math. Anal. Appl. 397, 522-528 (2013)

9. Simsek, Y: Generating functions of the twisted Bernoulli numbers and polynomials associated with their interpolation functions. Adv. Stud. Contemp. Math. 16(2), 251-278 (2008)

10. Simsek, Y: On $p$-adic twisted $q$-L-functions related to generalized twisted Bernoulli numbers. Russ. J. Math. Phys. 13(3), 340-348 (2006)

11. Faulhaber, J: Academia Algebræ, Darinnen die miraculosische Inventiones zu den höchsten Cossen weiters continuirt und profitert werden. Johann Ulrich Schonigs, Augsburg (1631)

12. Knuth, DE: Johann Faulhaber and sums of powers. Math. Comput. 61(203), 277-294 (1993)

13. Warnaar, SO: On the $q$-analogue of the sum of cubes. Electron. J. Comb. 11(1), N13 (2004) (electronic)

14. Fine, NJ: Basic Hypergeometric Series and Applications. Am. Math. Soc., Providence (1988)

15. Kim, T: A note on $p$-adic $q$-integral on $\mathbb{Z}_{p}$ associated with $q$-Euler numbers. Adv. Stud. Contemp. Math. 15(2), 133-137 (2007)

16. Kim, T: q-Volkenborn integration. Russ. J. Math. Phys. 9(3), 288-299 (2002)

17. Schlosser, $M:$-Analogues of the sums of consecutive integers, squares, cubes, quarts and quints. Electron. J. Comb. 11(1), R71 (2004) (electronic)

18. Carlitz, L: q-Bernoulli and Eulerian numbers. Trans. Am. Math. Soc. 76, 332-350 (1954)

19. Kim, T: On the q-extension of Euler and Genocchi numbers. J. Math. Anal. Appl. 326(2), 1458-1465 (2007)

20. Kim, T: On p-adic $q$-l-functions and sums of powers. J. Math. Anal. Appl. 329(2), 1472-1481 (2007)

21. Andrews, GE: On the theorems of Watson and Dragonette for Ramanujan's mock theta functions. Am. J. Math. 88 454-490 (1966)

22. Dragonette, L: Some asymptotic formulae for the mock theta series of Ramanujan. Trans. Am. Math. Soc. 72, 474-500 (1952)

23. Bringmann, K, Ono, K: The $f(q)$ mock theta function conjecture and partition ranks. Invent. Math. 165, $243-266$ (2006)

24. Hardy, GH, Seshu Aiyar, PV, Wilson, BM: Collected Papers of Srinivasa Ramanujan. Cambridge University Press, Cambridge (1927)

10.1186/1687-1847-2013-298

Cite this article as: Kim et al.: New approach to twisted $q$-Bernoulli polynomials. Advances in Difference Equations 2013, 2013:298 